\title{
Os estados brasileiros na transição demográfica: similaridades e características discriminantes
}

\section{Pascoal José Marion Filho', Lauana Rossetto Lazaretti ${ }^{2}$ Patricia Batistella ${ }^{3}$ e Felipe Orsolin Teixeira ${ }^{4}$}

\footnotetext{
1 Doutor em Economia Aplicada pela Escola Superior de Agricultura Luiz de Queiroz (ESALQ/ USP). Professor Titular do Departamento de Economia e Relações Internacionais. Professor do Programa de Pós-Graduação em Administração (PPGA) e do Programa de Pós-Graduação em Economia e Desenvolvimento (PPGE\&D) da Universidade Federal de Santa Maria (UFSM). E-mail: pascoaljmarion@yahoo.com.br

2 Mestranda em Economia e Desenvolvimento na Universidade Federal de Santa Maria (UFSM). E-mail: lauana.lazaretti@yahoo.com.br

3 Mestranda em Economia e Desenvolvimento na Universidade Federal de Santa Maria (UFSM). E-mail: patriciabatistella@rocketmail.com

4 Mestrando em Economia e Desenvolvimento na Universidade Federal de Santa Maria (UFSM). E-mail: felipeorsolin@bol.com.br
}

RESUMO: O tema transição demográfica vem se destacando nos debates acadêmicos e políticos por induzir a mudanças estruturais e levar ao redirecionamento de demandas da sociedade. O processo vem ocorrendo de maneira acelerada e heterogênea entre as regiões e dentro delas. O objetivo da pesquisa é identificar as similaridades na transição demográfica entre os estados brasileiros e agrupa-los pelas suas características socioeconômicas, nos anos de 2000 e 2010. Utiliza-se o método de análise fatorial para identificar os fatores comuns associados ao grau de relação, a técnica multivariada de cluster para agrupar os estados brasileiros e uma análise de discriminante para verificar quais variáveis possuem maior peso para a formação dos agrupamentos. Os dados utilizados são do IBGE (2016), DATASUS (2016) e PNUD (2016). Os resultados mostram que o processo da transição demográfica não é neutro, pois há relação entre as mudanças demográficas, econômicas e sociais, e que alguns estados brasileiros apresentam similaridades e características distintas no processo em 2000 e 2010, o que possibilita formar clusters de acordo com cada fase. Verifica-se também que nas regiões Sul e Sudeste a transição demográfica está em fase mais adiantada, e segue avançando para os demais estados brasileiros.

Palavras-chave: Transição Demográfica; Brasil; estados brasileiros; clusters.

The brazilian states in the demographic transition: similarities and discriminant characteristics

ABSTRACT: The demographic transition theme has been highlighting in academic and political debates for inducing structural changes and leading to the redirection of demands of society. The process has been occurring in an accelerated and heterogeneous way between regions and within them. The objective of the research is to identify the similarities in the demographic transition between Brazilian states and group them by their socioeconomic characteristics, in the years of 2000 and 2010. The factorial analysis method is used to identify the common factors associated to the degree of relation, the cluster multivariate technique to group the Brazilian states and a discriminant analysis to verify which variables have the greatest weight for cluster formation. The data used are from IBGE (2016), DATASUS (2016) and PNUD (2016). The results show that the demographic transition process is not neutral, since there is a relation between demographic, economic and social changes, and that some Brazilian states have different similarities and characteristics in the process in 2000 and 2010, which makes it possible to form clusters according to each phase. It is also verified that in the South and Southeast re- 
gions the demographic transition is at an advanced stage, and it continues to advance to the other Brazilian states.

Keywords: Demographic Transition; Brazil; Brazilian states; clusters.

\section{INTRODUÇÃO}

No início do século XX as preocupações populacionais da ONU e do Banco Mundial eram voltadas para a explosão demográfica e seus impactos sobre a oferta de alimentos e os recursos naturais. A preocupação mudou quando países mais desenvolvidos, como os da Europa, passaram a ter taxa de fecundidade baixa e crescimento populacional tendendo a zero. Essa característica vem se alastrando para outros continentes, trazendo consigo repercussões na estrutura etária e em suas economias (BRITO, 2008).

Com relação ao Brasil, nas últimas quatro décadas a estrutura social também passou por mudanças importantes. O País era considerado jovem, mas com a redução das taxas de fecundidade e de mortalidade, a distribuição etária da população ficou mais uniforme entre os grupos. As expectativas demográficas são de que a população de jovens se iguale a de idosos em 2050 (IBGE, 2016).

No entanto, essas mudanças não ocorrem parcimoniosamente entre os estados brasileiros (BRITO, 2008), pois há diversidade em suas formações históricas, que contribuem para desequilíbrios regionais e sociais. Como afirma o autor, a mudança na estrutura não é neutra, e esse processo social não altera apenas as variáveis demográficas, possui relação com variáveis econômicas e sociais, gerando efeitos positivos e negativos. $O$ processo de transição demográfica pode ser a causa da mudança em outros indicadores sociais e econômicos ou pode fazer parte de seus efeitos.
Em meio às diferenças regionais e as múltiplas variáveis envolvidas no processo de transição demográfica, o objetivo da pesquisa é identificar as similaridades na transição demográfica entre os estados brasileiros e agrupa-los pelas suas características socioeconômicas, nos anos de 2000 e 2010. Para tanto, utiliza-se suporte econométrico, envolvendo técnicas de análise multivariada: Fatorial, Cluster e Discriminante. A mudança no enquadramento regional permite determinar o ritmo e o local dos avanços econômicos e sociais, e cria condições para a elaboração de políticas específicas para reduzir a desigualdade.

Por fim, este trabalho está dividido em cinco seções, sendo a primeira delas esta introdução. Na segunda seção está o referencial teórico e na terceira os aspectos metodológicos. Na quarta seção são apresentados e discutidos resultados da pesquisa, e na quinta seção estão as considerações finais do estudo.

\section{REFERENCIAL TEÓRICO}

O debate sobre a relação entre a produção de alimentos e o crescimento populacional ficou marcado internacionalmente pela visão de Malthus (1986). O autor discordava das ideias sugeridas por Godwin, de que a terra seria capaz de produzir alimentos por muitos séculos para sustentar o aumento da população, uma vez que a população crescia a taxa geométrica e a produção de alimentos a taxa aritmética. Além disso, deduziu que com o maior crescimento da população vem a falta de alimentos, a 
miséria, a redução do preço do trabalho e o aumento do preço dos alimentos, o que reduz o vigor da humanidade e controla a sua expansão.

Segundo Bandeira (1996), a falta de perspectiva de longo prazo de Malthus foi superada, e os avanços tecnológicos foram os grandes responsáveis por criar uma realidade diferente daquela imaginada pelo autor. As técnicas implantadas na agricultura e na pecuária criaram as condições necessárias para o aumento da produtividade, afastando o efeito limitante causado pela falta de alimentos.

Para Galor e Weil (2000), além do Regime Maltusiano, a economia mundial passou ou deverá passar ao longo de sua história por mais dois regimes populacionais, o Regime Pós-Maltusino e o de Crescimento Moderno. O Regime Malthusiano é um processo de estagnação, em que há elevadas taxas de mortalidade e fecundidade, onde a tecnologia e a renda per capita não se alteram. No Regime Pós-Malthusiano, o nível tecnológico e a produtividade aumentam, e a taxa de mortalidade começa a diminuir. Além disso, a análise se concentra nas duas diferenças mais importantes entre esses regimes do ponto de vista macroeconômico: primeiro, no comportamento da renda per capita; e segundo, na relação entre o nível de renda per capita e a taxa de crescimento da população.

O Regime de Crescimento Moderno, para Galor e Weil (2000), caracteriza-se pelo crescimento da renda per capita e elevado nível tecnológico. Neste regime, tem-se uma relação negativa entre o nível de renda e a taxa de crescimento da população. Afirmam ainda que taxas de crescimento demográfico elevadas são notadas em países mais pobres, enquanto taxas tendendo a zero são encontradas em países mais ricos.

Porém, segundo Bloom, Canning e Sevilla (2001), enquanto o crescimento da população provoca efeitos negativos no crescimento econômico, as mudanças na estrutura etária da população, como o aumento da participação da população em idade ativa, aumenta o crescimento econômico. Alguns estudos teóricos, a exemplo do modelo de Solow (1956), consideram apenas o crescimento absoluto da população. No entanto, este não deve ser um parâmetro padrão de análise, pois a estrutura social se altera e muda o comportamento do consumo da população, o que também é um fator importante para o crescimento econômico.

Notestein (1953) acrescenta uma ideia evolucionária de transição demográfica, identificando três fases de desenvolvimento das populações: alto potencial de crescimento, transição de crescimento e declive incipiente. A primeira fase é marcada pelo rápido crescimento da população, favorecido pela elevada taxa de natalidade e queda da mortalidade. A segunda fase se caracteriza pela diminuição das taxas de fecundidade, provocando queda na taxa de natalidade mais acentuada que a de mortalidade, e desacelerando o ritmo de crescimento da população. Na terceira fase as taxas de natalidade e de fecundidade continuam baixas, proporcionando um crescimento populacional próximo de zero.

Levando em consideração que ainda não existe um consenso sobre as possíveis causas da queda na taxa de fecundidade, é importante destacar algumas suposições propostas por vários autores. Becker (1993) tratou os condicionantes econômicos de uma forma microeconômica e considerou que as crianças não podem ser equiparadas 
a um bem qualquer, pois geram custos pela utilização de bens e serviços, os quais se diferenciam em cada família. Esses custos irão pesar na decisão dos pais em ter filhos, assim constituindo uma espécie de função utilidade, na qual a demanda por crianças depende de seu custo e da renda familiar.

Para Caldwell (2004), existem dois tipos de regime de fertilidade, o primeiro é caracterizado pela inexistência de restrições a fecundidade e o segundo garante o ganho econômico com a restrição a fecundidade. $\mathrm{O}$ autor cita que mesmo em grandes famílias a escolha de ter filho é racional. No entanto, comenta que existem outros fatores que também influenciam na decisão do número de filhos, como os culturais, que geram mudanças institucionais e sociais. Entende ainda que o processo de industrialização trouxe consigo novas ideias e instituições. A difusão da nova cultura iniciada, principalmente, na Europa se estendeu para outras regiões, contribuído para a mudança na família tradicional, o desenvolvimento econômico e a queda nas taxas de fecundidade.

Também cabe destacar a explicação de Kirk (1996) sobre a queda na taxa de fecundidade. O autor aborda a retirada da ação das crianças como colaboradores econômicos e enfoca o custo crescente de sua criação, o qual surge em decorrência de três processos: i) do Estado, devido à fixação de idade mínima para trabalhar e à obrigatoriedade do ensino escolar; ii) das mudanças no mercado de trabalho, com aumento considerável da participação das mulheres; e, iii) devido a redefinição cultural das responsabilidades familiares com as crianças. Neste sentido, para Bloom, Canning e Sevilla (2001), foi após a Segunda Guerra Mundial que as taxas de fecundidade e mortali- dade diminuíram, devido aos avanços na saúde, no saneamento básico e na nutrição da população. Com isso, ocorreu um aumento da expectativa de vida, e com a redução do número de filhos houve a necessidade das famílias investirem mais em educação. Consequentemente, como ela é cara, a opção de um número menor de fiIhos viabiliza o processo e também contribui para a queda na taxa de fecundidade.

O estudo de Becker (2006) destaca que um nível maior de educação está relacionado a taxas de fecundidade menores. No entanto, o Brasil possui heterogeneidade entre as regiões e mesmo havendo uma tendência de convergência em longo prazo, as regiões ainda apresentam desigualdades entre si. Contudo, Paiva e Wajnman (2005) sugerem que a queda da fecundidade é estimulada pelo próprio desenvolvimento econômico da região, o que amplia o escopo para a análise e permite determinar padrões demográficos a partir de variáveis socioeconômicas, evidenciando as desigualdades regionais.

Kirk (1996) destaca ainda o papel do governo na queda da taxa de fecundidade e do índice de mortalidade. Segundo o autor, as políticas de promoção dos serviços públicos e de programas de planejamento familiar colaboram para o aumento da longevidade dos indivíduos. Além disso, algumas estratégias influenciaram no controle da natalidade e no aumento dos níveis de educação das mulheres e da saúde das crianças.

A transmissão de conhecimento e a comunicação entre os indivíduos, segundo Kirk (1996), também deve ser levada em conta ao analisar a queda dos níveis de fecundidade, pois o ser humano possui costumes, como o de compartilhar hábitos e 
rotinas. Além disso, o avanço das telecomunicações não deve ser tratado apenas de forma residual, mas sim como um agente de promoção ou retardo no controle dos níveis de fecundidade.

Em meio às discussões da ação do governo voltada para políticas de planejamento familiar, ganha espaço também o papel das mulheres neste processo de queda da fecundidade. Em 1994, com a Conferência Internacional de População e Desenvolvimento do Cairo, passa-se a dar ênfase no "empowerment" ${ }^{1}$ das mulheres, principalmente na sua decisão de escolha reprodutiva. Em 1999, Amartya Sen, juntamente com as novas dimensões que fazem parte do conceito de desenvolvimento, ressalta a importância da mulher como agente de mudança social, e que tanto o acesso à educação quanto ao mercado de trabalho está associado à queda dos níveis de fecundidade (PAIVA; WAJNMAN, 2005).

Em termos espaciais, Brito (2007) relata a importância da análise da estrutura etária da população a partir da região demográfica e dos diferentes níveis de renda. Em ambos os aspectos, o processo de transição demográfica é desigual, ou seja, há diferentes níveis de estrutura etária dentro dos grupos sociais e das regiões. Para o autor, o Brasil possui um nível de desigualdade relativamente alto, sendo que a pirâmide etária do grupo mais pobre da população possui taxa de fecundidade alta e o grupo de maior renda apresenta uma taxa de fecundidade baixa.

\section{METODOLOGIA}

${ }^{1}$ Empoderamento das mulheres para que participem integralmente de todos os setores da economia e em todos os níveis da atividade econômica (ONU, 2016).

\subsection{Análise fatorial}

Existem vários indicadores associados ao processo de transição demográfica. Na busca de análises mais parcimoniosas, reduz-se o número destes via análise fatorial, sendo possível descrever o comportamento do conjunto de $p$ variáveis por meio de um número menor de $r$ fatores comuns $(r<p)$.

Fávero et al. (2009), Mingoti (2005) e Hair et al. (2005) destacam a importância da análise fatorial para captar um número pequeno de fatores comuns representativos de um amplo conjunto de variáveis. A extração dos fatores é realizada pelo método de Análise de Fatores Comuns (AFC), que utiliza a maior variância total explicada pelo conjunto de indicadores e procura agrupar as variáveis mais correlacionadas, reduzindo significativamente a dimensão sem perder os aspectos essenciais das variáveis. 0 método Varimax é utilizado na rotação ortogonal dos fatores e, com isso, busca-se identificar uma variável com alta carga fatorial com um único fator (FÁVERO et al., 2009). Segundo Lima (2011), o método de rotação de fatores possibilita a melhor interpretação das cargas fatoriais, ou seja, identificar as variáveis que melhor se relacionam com os fatores.

O modelo de análise fatorial ortogonal consiste em um conjunto de variáveis com vetores de médias, matriz de variância e covariância e matriz de correlações. A variação total explicada pode ser separada em três conjuntos (Equação 1):

Variação Total = Comunalidade +

Unicidade + Erro

Sendo:

Comunalidade: os fatores comuns, que in- 
fluenciam duas ou mais variáveis;

Unicidade: os fatores específicos, que contribuem para a variação de uma única variável.

Assim, considerando que a unicidade e o erro constituem $\xi$ e as variáveis estão padronizadas, o modelo de análise fatorial relaciona linearmente as variáveis, os fatores comuns, a unicidade e o erro (Equação 2):

$$
\begin{array}{cc}
x_{1}=a_{11} F_{1}+a_{12} F_{2}+a_{13} F_{3} \\
& +\cdots+a_{1 r} F_{r}+\varepsilon_{1} \\
\vdots & \\
\vdots & \\
x_{r}=a_{p 1} F_{1} & +a_{p 2} F_{2}+a_{p 3} F_{3} \\
& +\cdots+a_{p r} F_{r}+\varepsilon_{1}
\end{array}
$$

Representando em termos matriciais (Equação 3):

$$
\begin{aligned}
\boldsymbol{X}_{p x 1}=\boldsymbol{A}_{p x r} & \boldsymbol{F}_{r x 1} \\
& +\boldsymbol{\varepsilon}_{p x 1}
\end{aligned}
$$

Em que:

$\boldsymbol{X}=$ Vetor de variáveis originais;

$\boldsymbol{F}=$ Vetor de fatores comuns;

$\boldsymbol{A}=$ Matriz de cargas fatoriais;

$\boldsymbol{\varepsilon}=$ Vetor de erros aleatórios;

$r$ (número de fatores) $<p$ (número de variáveis).

Este modelo tem como objetivo explicar o comportamento das variáveis em função dos fatores comuns e do termo de erro (unicidade + termo de erro). 0 modelo ortogonal pressupõe que a esperança do erro seja zero, todos os fatores têm média zero, variância 1 (um) e não são correlacionados. Os erros podem ter variâncias diferentes e não correlacionados e os fatores comuns são independentes dos específicos e dos erros.

$\mathrm{Na}$ aplicação da análise fatorial é possível utilizar a matriz de variâncias e de covariâncias ou a matriz de correlações, sendo que a matriz de variâncias e covariâncias das variáveis padronizadas é igual à matriz de correlações das variáveis originais. No trabaIho, utiliza-se a matriz de correlações, conforme sugerido por Hair et al. (2005). A matriz de correlações pode ser decomposta em duas partes, denominadas de comunalidade e unicidade (Equação 4). Assim,

$h_{j}^{2}+\varphi_{j}=1$, ou comunalidade + unicidade $=1$

A comunalidade é a parcela de variância que os fatores conseguem explicar, e a unicidade é a parcela que não pode ser explicada. O intuito da análise fatorial é a determinação destas duas matrizes, que reproduzem a matriz de correlações com um número menor de variáveis que as originais. A estimação das cargas fatoriais utilizada no estudo é realizada pelo método dos componentes principais, que possui como base o uso das raízes características e dos vetores característicos para a determinação das matrizes.

A identificação das variáveis utilizadas na análise foi obtida através da revisão teórica sobre o tema. Em um conjunto mais amplo de variáveis, as utilizadas no trabalho são descritas abaixo, visto que algumas (como, por exemplo, Taxa de Dependência Total e PIB agropecuário) não podem ser utilizadas, pois possuem autocorrelação com outras variáveis. Todas as variáveis utilizadas na análise fatorial foram padronizadas e estão em percentual: 
$\mathrm{X}_{1}=$ Razão de dependência Jovem ${ }^{2}$;

$\mathrm{X}_{2}=$ Razão de dependência Idosa ${ }^{3}$;

$\mathrm{X}_{3}=$ Percentual do Produto Interno Bruto da indústria no estado $i$;

$\mathrm{X}_{4}=$ Percentual do Produto Interno Bruto dos serviços no estado $i$;

$\mathrm{X}_{5}=$ População em Idade Ativa;

$\mathrm{X}_{6}=$ Taxa de crescimento da população;

$\mathrm{X}_{7}=$ Percentual de mães chefes de família sem ensino fundamental e com filho menor de idade;

$\mathrm{X}_{8}=$ Taxa de envelhecimento;

$\mathrm{X}_{9}=$ Taxa de mortalidade infantil;

$\mathrm{X}_{10}=$ Probabilidade de sobrevivência até 60 anos;

$\mathrm{X}_{11}=$ Taxa de fecundidade total;

$\mathrm{X}_{12}=$ Percentual da população de 25 anos ou mais com ensino fundamental completo; $\mathrm{X}_{13}=$ Percentual da população de 25 anos ou mais com ensino médio completo;

$\mathrm{X}_{14}=$ Percentual da população de 25 anos ou mais com ensino superior completo; $\mathrm{X}_{15}=$ Expectativas de anos de estudo ${ }^{4}$.

As variáveis utilizadas correspondem aos anos de 2000 e 2010, e foram extraídas do DATASUS (2016), PNUD (2016) e do IBGE (2016). O padrão de medida são os estados brasileiros.

Para testar a adequação da análise são utilizados os testes de KMO e Bartlett. O

${ }^{2} R D J=\frac{n \text { o de pessoas de } 0 \text { a } 14 \text { anos }}{n \div \text { de pessoas em idade ativa (15-64 anos })}$

${ }^{3} R D I=\frac{n \text { o de pessoas de } 65 \text { anos ou mais }}{n \circ \text { de pessoas em idade ativa (15-64 anos) }}$

${ }^{4}$ É uma adaptação metodológica da métrica aplicada no IDH Global. Considera 12 anos de estudo como o máximo da educação formal (ensino básico) e ajusta estes valores para a repetência. Ou seja, considera apenas a adequação da frequência escolar até os 18 anos de idade. No caso de um fluxo escolar ideal, em que todas as pessoas ingressam aos 6 anos no ensino fundamental e não há repetência ou abandono ao longo do ensino básico, esse indicador assumiria o valor de 12 anos (PNUD, 2016). teste KMO possibilita verificar a correlação entre as variáveis, onde 0 (zero) indica que ela não existe e 1 (um) que ela ocorre. 0 teste de esfericidade de Bartlett testa a hipótese nula da matriz de correlações ser identidade (correlação zero entre as variáveis). Quando rejeitada, há correlação entre as variáveis e o modelo pode ser utilizado. Quanto aos escores fatoriais, o método utilizado é o Bartlett.

\subsection{Análise de cluster}

A análise de cluster visa agrupar os estados brasileiros por meio de suas semelhanças quanto aos atributos demográficos que possuem e suas relações com outras variáveis. Para isso, foram utilizados dados censitários dos anos de 2000 e 2010, com variáveis dos estados brasileiros $\left(X_{1}-X_{15}\right)$ e relacionadas na seção 3.1 da análise fatorial.

Hair et al. (2005) consideram a análise de agrupamentos (clusters) como uma técnica multivariada, que possui o intuito de gerar objetos com base nas propriedades que os mesmos possuem. Segundo Mingoti (2005), os agrupamentos tendem a dividir os elementos da amostra a partir da homogeneidade dentro dos grupos e da heterogeneidade entre eles. Para Hair et al. (2005), a constituição de algoritmo para os grupos pode ser feita através de critérios como o de similaridade ou dissimilaridade (parecença). No trabalho, o critério de parecença utilizado é o da distância Euclidiana Quadrática.

Segundo Hair et al. (2005), essa medida de distância possui propriedades como: base econométrica interessante, invariante com relação à transformação de origem e a ortogonalidade, e não invariante com relação à transformação de escala e não orto- 
gonal. É uma das medidas de distâncias mais utilizadas.

Para a construção dos agrupamentos, podem-se utilizar técnicas hierárquicas e não hierárquicas. No caso do estudo, a técnica utilizada é a hierárquica. Para Hair et al. (2005), esse método aglomerativo faz com que a cada novo algoritmo os elementos da análise vão se agrupando, e no fim haverá apenas um grupo que envolve todos os elementos. Para a aplicação desta técnica o método utilizado é o de Ward.

Segundo Lima (2011, p.9), o Método Ward:

No processo aglomerativo, à medida que se agrupa o nível de similaridade diminui. Em cada passo do agrupamento ocorre diminuição de variabilidade entre os grupos e aumento dentro dos grupos. O método Ward se baseia na mudança de variação que ocorre de um estágio para outro. Este método tende a formar grupos com maior homogeneidade interna.

Para determinar o número de grupos foi utilizada a regra da parada, a partir da análise do cronograma de aglomeração ( $\mathrm{Ag}$ glomeration Schedule), em que saltos grandes na distância entre um estágio e outro indicam possíveis pontos de corte, conforme estudos de Fávero et al. (2009). Utilizase na análise das estatísticas Pseudo $T^{2}$ e Pseudo F.

\subsection{Análise de discriminante}

Entre as técnicas de análise multivariada de dados está a análise de discriminante. Tal método tem por objetivo separar e classificar observações em populações previamente definidas. Discriminar é encontrar funções capazes de explicar o máximo de separação entre os grupos levando em consideração as diversas variáveis. Para a classificação, utilizam-se as funções obtidas para alocar as novas observações nos grupos que já foram estabelecidos (HAIR et al., 2005).

De acordo com Hair et al. (2005), o primeiro passo para a aplicação deste método é a definição dos grupos. Levando em consideração suas características gerais, a definição do grupo deve ser embasada na problemática e objetivo do estudo. Dessa forma, pretende-se obter um número mínimo de observações incorretas. Com isso, evitase afirmar que um elemento que pertence ao grupo $X$, na verdade, devido a suas características, deveria pertencer ao grupo $Y$.

$\mathrm{Na}$ análise de discriminante é necessário possuir diversas populações com " $\mathrm{g}$ " amostras, descritas pelas mesmas " $p$ " variáveis. $\mathrm{Na}$ análise de cluster, a qual busca formar " $k$ " grupos homogêneos na amostra, o método da análise de discriminante parte de um número já definido de grupos com amostra de populações diferentes, não busca defini-los, mas sim interpretar as variáveis (HAIR et al., 2005).

As funções que são obtidas na análise de discriminante são capazes de separar grupos e determinar combinações lineares das variáveis $\mathrm{X}\left(Y=a^{\prime} X\right)$, que definem os elementos dos grupos e determinam os coeficientes $a_{1}, a_{2}, \ldots, a_{p}$. O método mais utilizado para se obter os coeficientes é o método Fisher.

Supondo que sejam duas populações, $\pi_{1}$, $\pi_{2}$ e $p$ variáveis medidas nestas amostras, tem-se:

$\mu_{1}=$ vetor de médias de $\pi_{1}$; 
$\mu_{2}=$ vetor de médias de $\pi_{2}$;

$\Sigma_{1}=$ matriz de variâncias e covariâncias de

$\pi_{1}$

$\sum_{2}=$ matriz de variâncias e covariâncias de $\pi_{2}$.

Obtendo as amostras de tamanhos $n_{1}$ de $\pi_{1}$ e $n_{2}$ de $\pi_{2}$, é possível definir as estimativas amostrais:

$\overline{X_{1}}=$ vetor $p x 1$ de médias da amostra de $\pi_{1}$; $\overline{X_{2}}=$ vetor $p x 1$ de médias da amostra de $\pi_{2}$; $S_{1}=$ matriz de variâncias e covariâncias da amostra $\pi_{1}$;

$S_{2}=$ matriz de variâncias e covariâncias da amostra $\pi_{2}$.

Dessa forma, quanto mais longe forem as médias das amostras, maior é a probabilidade de serem diferentes.

Presumindo que $\sum_{1}=\sum_{2}=\sum$, a matriz comum $\sum$ é estimada pela matriz de variâncias e covariâncias conjunta, representada pela Equação 5:

$S_{p x p}=\frac{\left(n_{1}-1\right) S_{1}+\left(n_{2}-1\right) S_{2}}{n_{1}+n_{2}-2}$

O resultado $\left(S_{p x p}\right)$ representa um estimador não tendencioso de $\sum$. Segundo Lima (2011), o princípio básico do método de Fisher é determinar uma ou mais combinações lineares possíveis das variáveis analisadas, sendo que o número de funções de discriminantes irá depender do número de grupos, ou seja, se possuir $g$ grupos serão calculadas $g-1$ funções. Tais funções serão indicadas de acordo com sua relevância, a primeira função será a que mais discrimina os grupos, e assim sucessivamente.

Os testes estáticos utilizados para este método são: teste de igualdade de médias entre os grupos, Testes Box's M e Wilks' Lambda.

\section{RESULTADOS E DISCUSSÃO}

\subsection{Os Fatores Comuns}

A técnica proporciona agrupamentos que explicam a maior variabilidade dos dados. No caso da transição demográfica, a qual possui relação com diversos indicadores, à técnica de análise fatorial contribui para determinar um padrão de transição demográfica dos estados brasileiros nos anos de 2000 e 2010. No Quadro 1 são apresentados os fatores e as suas respectivas variáveis.

Quadro 1 - Determinação dos fatores comuns para as quinze variáveis econômicas e sociais de 2000 e 2010

\begin{tabular}{|c|l|c|}
\hline \multicolumn{3}{|c|}{ Censo Demográfico 2000} \\
\hline Fator & Especificação & Variáveis \\
\hline$F_{1}$ & Idade ativa e educação & $X_{5}, X_{12}, X_{13} X_{14} \mathrm{e} X_{15}$ \\
\hline$F_{2}$ & Características populacionais & $X_{1}, X_{2}, X_{6}, X_{7}$ e $X_{8}$ \\
\hline$F_{3}$ & Indicadores socioeconômicos & $X_{3}, X_{4}, X_{9}, \mathrm{X}_{10}$ e $\mathrm{X}_{11}$ \\
\hline \multicolumn{3}{|l|}{ Censo Demográfico 2010 } \\
\hline Fator & Especificação & Variáveis \\
\hline$F_{1}$ & $\begin{array}{l}\text { Transição demográfica e } \\
\text { características sociais }\end{array}$ & $\begin{array}{c}X_{1}, X_{2}, X_{5}, X_{6}, X_{8}, \mathrm{X}_{10}, \mathrm{X}_{11} \\
e X_{15}\end{array}$ \\
\hline$F_{2}$ & Educação & $X_{7}, \mathrm{X}_{9}, X_{12}, X_{13}$ e $X_{14}$ \\
\hline$F_{3}$ & Produtividade & $X_{3}$ eX \\
\hline
\end{tabular}

Fonte: Resultados do trabalho.

Ao analisar os dados do censo demográfico de 2000, constata-se maior intensidade do fator 1 (idade ativa e educação) para o Distrito Federal, Rio de Janeiro, São Paulo e Rio Grande do Sul. Os mesmos possuem um percentual da população em idade ativa (PIA) entre os mais altos do País, 68,30\%, $67,40 \%, 67,58 \%$ e $66,73 \%$, respectivamente. Tais regiões também possuem elevados índices educacionais, ou seja, há um maior número de pessoas que possuem ensino fundamental, médio e superior, comparado 
aos outros estados. Ao mesmo tempo em que estes estados possuem características semelhantes quanto à educação e a PIA, verifica-se que há correlação entre as variáveis sociais e demográficas.

No outro extremo estão os estados que possuem os mais baixos índices do fator 1 : Piauí, Alagoas e Maranhão, com uma PIA de $60,73 \%, 59,93 \%$ e $57,83 \%$, respectivamente. Quando se compara o nível de educação entre os estados, observa-se que o Rio de Janeiro, o mais intensivo, chega a ter o dobro do índice do estado que possui o menor indicador, o Maranhão, o que demonstra um elevado nível de desigualdade no Brasil, também observado por Brito (2007).

O fator 2 (características populacionais) possui maior indicador no Amapá, Roraima e Acre. Estes estados ainda possuem altas taxas da razão de dependência de jovens (RDJ) e de crescimento da população, o que os coloca na primeira fase de desenvolvimento da população de Notestein (1953). Os estados do Rio Grande do Sul, Rio de Janeiro e Paraíba possuem, na ordem, as mais baixas taxas de crescimento da população e da PIA, ou seja, estão na última fase identificada pelo autor (declive incipiente).

Quanto ao fator 3 (indicadores socioeconômicos), os maiores índices se concentram nos estados do Maranhão, Alagoas e Amapá, onde as taxas de fecundidade e mortalidade são elevadas. Em situação oposta está Mato Grosso, Roraima e Santa Catarina, os quais apresentam os menores níveis desse fator.

Em relação ao ano de 2010, para o mesmo conjunto de variáveis analisadas anteriormente, percebe-se que os fatores sofreram alterações em suas formações. O fator 1 (transição demográfica e características sociais) é mais expressivo para Santa Cata- rina, Tocantins e São Paulo. Estes estados possuem um elevado percentual de pessoas em idade ativa, bem como alta expectativa de anos de estudo. No outro extremo, encontram-se Mato Grosso, Minas Gerais e Acre, nesses prevalece um alto nível de crescimento da população.

O fator 2 (educação), em 2010, agrega os estados de Sergipe e Paraíba pelos baixos níveis educacionais da população de 25 anos ou mais, com ensino fundamental, médio e superior. Entretanto, Rondônia e Amapá, que também apresentam baixo nível de educação, possuem elevado percentual de mães chefes de família sem ensino fundamental e com filho menor de idade, e as maiores taxas de mortalidade infantil.

Os dados que compõem o fator 3 (produtividade), no ano de 2010, estão relacionados aos PIB industrial e de serviços. O Estado de Santa Catarina se destaca por apresentar a maior participação da indústria no PIB total. Por outro lado, Sergipe possui baixo percentual da indústria no seu PIB e, também, caracteriza-se por baixo nível deste fator.

Ao comparar as duas análises fatoriais, verifica-se que a relação entre os fatores demográficos e as demais variáveis socioeconômicas apresentaram diferentes correlações para a formação dos fatores. Tais resultados corroboram com o estudo realizado por Brito (2008), o qual define a transição demográfica como um processo que não é neutro e pode se diferenciar entre regiões e no decorrer dos anos.

Para assegurar a consistência dos fatores foram realizados os testes de KMO e de Bartlett (Tabela 1). O índice de KMO aponta a existência de correlações entre as variáveis, sendo de 0,63 em 2000 e de 0,65 em 2010, o que indica que os dados são consis- 
tentes. O teste de Bartlett ( $p$-valor: 0,000) confirma que há correlação entre as variáveis (FÁVERO et al., 2009; HAIR et al., 2005).

Tabela 1 - Teste de Kaiser-Meyer-Olkin e Bartlett's

Teste de KMO and Bartlett's 2000

Kaiser-Meyer-Olkin Measure of Sampling Adequacy.

Bartlett's Test of

Sphericity

\begin{tabular}{|l|r|}
\hline Approx. Chi-Square & 639,78 \\
\hline Df & 91 \\
\hline Sig. & 0,000 \\
\hline
\end{tabular}

Teste de KMO and Bartlett's 2010

Kaiser-Meyer-Olkin Measure of Sampling Adequacy.

Bartlett's Test of

Sphericity

Approx. Chi-Square

Sig.

Fonte: Resultados do trabalho.

Como os testes realizados foram favoráveis à aplicação da análise fatorial, passouse a estimativa dos fatores comuns rotacionados pelo método Varimax. Para a definição do número de fatores há diferentes critérios de extração, mas neste estudo optouse por utilizar os componentes que captam pelo menos $70 \%$ da variância acumulada. Sendo assim, a partir dos quinze indicadores analisados foi possível extrair três fatores comuns para os dois períodos, que foram capazes de captar $78,46 \%$ da variância das variáveis em 2000 e 76,46\% em 2010.

\subsection{Os clusters e as características dos es- tados}

A transição demográfica brasileira não pode ser caracterizada como um processo neutro e isolado (BRITO, 2008). Fatores históricos e institucionais também devem ser levados em conta quando tratadas as diversidades regionais do País. Desta forma, buscando não excluir as peculiaridades de cada estado, o estudo trata-os de forma independente.

O estudo analisa a situação de 26 estados e o Distrito Federal, uma análise descri- tiva dos dados poderia limitar a análise e a extração de resultados importantes. Visando maximizar a homogeneidade dentre os grupos e maximizar a heterogeneidade entre eles, a análise multivariada de cluster se faz pertinente. Com isso, agrupam-se os estados que possuem similaridades (características comuns) entre si com respeito às variáveis selecionadas em 2000 (sete aglomerações) e 2010 (quatro aglomerações).

Dentre as principais evidências da formação dos clusters em 2000 foi possível constatar diferentes processos de transição demográfica. Os estados de Amapá e Acre formam o cluster 1 e apresentam uma alta taxa de crescimento da população jovem, contribuindo para uma transição demográfica tardia.

O agrupamento 2 (Rondônia, Rio Grande do Norte, Piauí e Sergipe) se caracteriza por possuir um percentual da população em idade ativa (PIA) próximo de $61 \%$ do total, com baixa mortalidade infantil e expectativa de anos de estudo mediana.

Os estados que constituem o cluster 3 (Maranhão, Alagoas, Paraíba, Pernambuco, Tocantins, Ceará e Bahia) possuem cerca de $17 \%$ de mães sem ensino fundamental, mortalidade infantil alta e percentual de pessoas com ensino fundamental, médio e superior baixos.

O cluster 4 (Amazonas, Pará e Roraima) é caracterizado pela razão de dependência de jovens alta e de idosos baixa. O percentual da população entre 15 a 65 anos de idade está entre os mais baixos dos estados brasileiros, ficando atrás apenas dos estados do cluster 1 (Amapá e Acre), e a taxa de crescimento da população é elevada.

No cluster 5 (Mato Grosso do Sul, Minas Gerais, Goiás, Espírito Santo, Mato Grosso e Santa Catarina) a RDJ é mediana, a PIA é 
alta e a taxa de mortalidade infantil é a mais baixa entre os estados. Já o cluster 6 (Distrito Federal) representa um outlier da variável percentual do PIB serviços e um valor baixo no PIB industrial.

Entretanto, os estados que compõem o cluster 7 (Rio Grande do Sul, Rio de Janeiro, São Paulo e Paraná) já estão em um processo avançado de transição demográfica [a terceira fase de Notestein (1953)], e a população de jovens e idosos tendem a se igualar no decorrer dos próximos anos. Nesses estados o percentual de população jovem caiu de forma acentuada nas últimas décadas e a taxa de fecundidade é baixa.

Quando se observa os resultados dos agrupamentos para o ano de 2010, constatase uma formação de 4 clusters. Algumas unidades se agruparam com estados que no período anterior apresentavam características muito distintas, como é o caso do Distrito Federal.

O primeiro cluster (Acre, Bahia, Ceará, Maranhão, Paraíba, Pernambuco, Piauí e Sergipe) é caracterizado por elevado percentual do PIB de serviços, alta taxa de mães chefes de família sem ensino fundamental e probabilidade de sobrevivência até 60 anos.

É correto afirmar que o agrupamento 2 (Espírito Santo, Goiás, Mato Grosso, Mato Grosso do Sul, Minas Gerais, Paraná, Rio de Janeiro, Rio Grande do sul, Rio Grande do Norte, Rondônia, Santa Catarina, São Paulo e Tocantins), o qual inclui o maior número de estados, caracteriza-se principalmente por possuir afinidades com respeito as variáveis educação elevada, taxa de fecundidade baixa e mortalidade infantil inferior aos demais estados, sendo estas últimas variáveis relevantes ao tratar do processo de transição de demográfica.
O cluster 3 (Alagoas, Amazonas e Pará) possui os percentuais mais altos relativos ao PIB da indústria, bem como do percentual da população em idade ativa. $\mathrm{O}$ quarto agrupamento (Amapá, Distrito Federal e Roraima), como na análise de 2000 , possui vinculo com o percentual do PIB de serviços elevado, chegando a 93\% no Distrito Federal, $86 \%$ no Amapá e $82 \%$ em Roraima.

Ao comparar a formação dos clusters de 2000 e 2010, constata-se uma mudança na formação dos grupos, e esta decorre do processo de transição demográfica acelerado no Sul e Sudeste, que se espalha para outras regiões. Com estes, também se espraiam variáveis sociais mais favoráveis nestas regiões, como maior educação, menor mortalidade infantil e elevada probabilidade de sobrevivência. Quanto as variáveis econômicas, especificamente o PIB dos estados, ainda há expressiva diversidade em relação aos setores que os compõem.

A formação dos clusters quando associadas às variáveis que representam fatores demográficos, econômicos e sociais, mostram que a transição demográfica não deve ser considerada um processo isolado. Comparando os resultados, verifica-se que a estrutura etária da população ao sofrer alterações, provoca mudanças econômicas e sociais. Brito (2008) salienta a importância de políticas públicas, pois o crescimento populacional se distribui entre as várias faixas de renda e o crescimento mais acelerado se concentra nas famílias mais pobres. Assim, os benefícios demográficos estão fortemente ligados com a situação social brasileira.

As políticas brasileiras ainda devem levar em consideração os desequilíbrios regionais, visto que o Brasil é marcado por possuir heterogeneidade entre as regiões e 
estados. Brito (2008) ressalta as diferentes etapas da transição demográfica nos estados brasileiros, e elas estão de acordo com os resultados encontrados no trabalho, já que no Nordeste a razão de dependência é maior, enquanto em São Paulo e no extremo Sul são menores.

Não há um critério preestabelecido para a definição do número de cluster. No estudo, optou-se por testes pseudo- $T^{2}$ e pseudo- $F$ de Calinski-Harabasz. O maior valor do teste pseudo-F e o menor valor do pseudo$T^{2}$ indicam grupos mais distintos, sendo com isso possível manter as peculiaridades de cada grupo e preservar a homogeneidade entre eles.

\subsection{Análise de Discriminante}

Os coeficientes da função discriminante apresentados na Tabela 2 retratam valores que se aplicam a discriminação das variáveis após serem padronizadas pela covariância dentro do grupo. Tais coeficientes são apropriados para interpretar a importância e a relação das variáveis dentro das funções discriminantes.

Tabela 2 - Funções discriminantes dos agrupamentos para o ano de 2000

\begin{tabular}{lrrrrrr}
\hline Variáveis & Função 1 & Função 2 & Função 3 & Função 4 & Função 5 & Função 6 \\
\hline RDJ & 23,4808 & $-13,5922$ & $-10,7813$ & 3,2920 & $-7,4020$ & 1,3100 \\
RDI & 20,8748 & $-2,2744$ & $-2,8781$ & 1,5390 & $-3,5053$ & 2,0145 \\
PIB industri- & $-1,5807$ & 0,2160 & 0,8278 & $-0,1490$ & 1,2464 & $-0,0720$ \\
al & & & & & & \\
PIB serviços & $-2,1129$ & $-0,9841$ & 0,6710 & $-0,8036$ & 0,6261 & $-0,4821$ \\
PIA & 23,5711 & $-11,6287$ & $-9,6818$ & 3,1240 & $-7,8385$ & 1,2891 \\
$\begin{array}{l}\text { Taxa de } \\
\text { crescimento }\end{array}$ & $-0,6689$ & $-0,1509$ & $-1,0993$ & 1,0051 & $-1,7066$ & 0,6684 \\
$\begin{array}{l}\text { Percentual } \\
\text { de mães }\end{array}$ & $-0,5208$ & $-0,1024$ & $-0,8414$ & 0,0481 & $-0,5900$ & 0,0350 \\
chefes* & & & & & & \\
$\begin{array}{l}\text { Taxa de } \\
\text { envelheci- }\end{array}$ & $-13,1574$ & $-1,9066$ & $-2,7322$ & $-0,1900$ & $-0,7705$ & $-1,7410$ \\
mento de & 0,9163 & $-0,3623$ & $-1,7947$ & $-0,2607$ & 0,1001 & 0,2663 \\
$\begin{array}{l}\text { Taxa de } \\
\text { mortalidade }\end{array}$ & $-0,4626$ & $-1,1040$ & $-0,5783$ & 0,5705 & $-0,4399$ & 0,1390 \\
$\begin{array}{l}\text { Prob. } \\
\text { sobrevivên- } \\
\text { cia }\end{array}$ & & & & & & \\
$\begin{array}{l}\text { Taxa de } \\
\text { fecundidade }\end{array}$ & $-0,5202$ & 0,7362 & 0,1127 & 0,4169 & $-0,8600$ & $-0,3156$ \\
Ensino & $-2,7522$ & 2,3701 & $-4,7628$ & $-0,0069$ & 1,7701 & $-3,0559$
\end{tabular}

\begin{tabular}{|c|c|c|c|c|c|c|}
\hline $\begin{array}{l}\text { fundamental } \\
\text { Ensino } \\
\text { médio }\end{array}$ & 3,5847 & $-2,1668$ & 4,6417 & $-0,3148$ & $-0,3966$ & 2,4780 \\
\hline $\begin{array}{l}\text { Ensino } \\
\text { superior }\end{array}$ & 0,6247 & $-0,7972$ & $-0,6538$ & 0,4984 & $-0,3289$ & 0,4893 \\
\hline $\begin{array}{l}\text { Expectativa } \\
\text { de estudo }\end{array}$ & 2,0474 & 0,6283 & 1,1360 & $-0,3915$ & $-0,0440$ & $-0,1705$ \\
\hline
\end{tabular}

Na análise de cluster para o ano de 2000 foram obtidos 7 agrupamentos, e por isso foram geradas seis funções discriminantes. Como a sétima função discriminante é responsável por uma porcentagem pequena da variância, ignoram-se os coeficientes desta função ao avaliar a importância das variáveis.

A primeira função discriminante evidência a importância das variáveis Razão de Dependência de Jovens (RDJ) e Razão de Dependência de Idosos (RDI) para a formação do primeiro cluster. As funções dois, três, quatro e cinco possuem um coeficiente alto para as variáveis RDJ e para População em Idade Ativa (PIA), sendo os principais responsáveis pela formação destes clusters. As variáveis, percentual de pessoas com ensino fundamental e ensino médio contribuem para a formação do sexto agrupamento.

Na análise de cluster para o ano de 2010 foram extraídos quatro agrupamentos, e o que resultou em três funções discriminantes a serem analisadas. Na Tabela 3 são apresentadas as funções para os grupos formados a partir do Censo Demográfico de 2010.

Tabela 3 - Funções discriminantes dos agrupamentos para o ano de 2010

\begin{tabular}{lrrr}
\hline \multicolumn{1}{c}{ Variáveis } & \multicolumn{1}{c}{ Função 1 } & Função 2 & \multicolumn{1}{c}{ Função 3 } \\
\hline RDJ & $-3,6667$ & $-11,9381$ & 8,5324 \\
RDI & $-6,9453$ & $-4,6773$ & $-1,2575$ \\
PIB industrial & 1,0148 & $-1,3688$ & $-1,0956$ \\
PIB serviços & 2,9761 & $-0,7267$ & $-0,3441$ \\
PIA & $-3,3198$ & $-10,2132$ & 6,8475 \\
Taxa de crescimento & 1,4472 & $-0,2926$ & $-0,2596$
\end{tabular}




\begin{tabular}{lrrr} 
Percentual de mães chefes* & $-0,2599$ & $-0,8268$ & 0,8997 \\
Taxa de envelhecimento & 4,4341 & 1,1545 & 5,2969 \\
Taxa de mortalidade & 0,3530 & $-0,4933$ & $-0,6358$ \\
Prob. de sobrevivência & $-0,6324$ & $-0,7548$ & $-0,0139$ \\
Taxa de fecundidade & $-0,4232$ & 0,1856 & $-0,1382$ \\
Ensino fundamental & 5,1394 & 1,0209 & $-0,6734$ \\
Ensino médio & 5,1394 & 1,0090 & $-0,0924$ \\
Ensino superior & $-0,8327$ & $-1,6632$ & 0,0240 \\
Expectativa de estudo & 0,3406 & 0,9101 & 0,5254 \\
\hline
\end{tabular}

Fonte: Resultados da pesquisa.

Nota: * Percentual de mães chefes de família sem ensino fundamental e com filho menor de idade.

A primeira função de discriminante para o ano de 2010 apresenta alto coeficiente para as variáveis Razão de Dependência Idosa (RDI) e percentual da população com ensino fundamental completo. As funções dois e três possuem correlação com Razão de Dependência de Jovens e População em Idade Ativa.

As principais variáveis que influenciaram a formação dos agrupamentos nos anos de 2000, também se mantiveram para a formação dos grupos no ano de 2010. Essas variáveis são principalmente atreladas a características demográficas e de educação. Tais resultados vêm a confirmar o que já havia sido evidenciado na análise preliminar para a formação dos clusters.

Assim, a transição demográfica não pode ser analisada como um processo neutro, nem mesmo homogêneo, pois os estados estão em diferentes fases de desenvolvimento das populações, conforme definido por Notestein (1953). Os benefícios gerados por ela podem ser positivos do ponto de vista estrutural, mas do ponto de vista social preserva as desigualdades. Neste sentido, as políticas sociais são importantes, pois a transição demográfica é um processo que gradativamente vai perdendo espaço social e possibilita que nos grupos mais altos da sociedade os resultados sejam mais eficazes (BRITO, 2008).

\section{CONSIDERAÇÕES FINAIS}

A transição demográfica é um processo que afeta o ambiente socioeconômico regional e impacta no desenvolvimento e no bem estar da população. Na pesquisa, é possível afirmar que existe uma relação entre as variáveis demográficas, econômicas e sociais nos dois censos demográficos (2000 e 2010), mas ela se modifica ao longo do tempo em decorrência da estrutura etária.

No censo de 2000, as variáveis demográficas possuem correlações principalmente com a educação, e no ano de 2010 os indicadores populacionais se agrupam com outras variáveis socioeconômicas, como a probabilidade de sobrevivência e a expectativa de vida. Desta forma, observa-se a interação dos diversos elementos que compõem a análise.

Através da análise de cluster é possível identificar a transição demográfica como um processo não isolado, e que ocorre de diferentes formas nos estados brasileiros. No ano de 2000, foi possível extrair sete agrupamentos e identificar que os estados do Amapá e Acre possuem um processo de transição demográfica tardia, enquanto Rio Grande do Sul, Rio de Janeiro, São Paulo e Paraná estão mais avançados nesse processo.

No ano de 2010, formaram-se quatro clusters e foi possível observar que as regiões Sul e Sudeste, as mais avançadas na transição demográfica, continuaram evoluindo e influenciaram os demais estados próximos, no que tange a semelhança das características populacionais e condições socioeconômicas. Assim, os resultados indicam para uma possível convergência no processo de transição demográfica entre os estados brasileiros. 
Diante da diversidade, das características históricas e peculiaridades econômicas e sociais dos estados brasileiros, tornam-se relevantes políticas governamentais que possam amenizar os impactos negativos sobre as faixas mais vulneráveis da população. Para os estados com um processo de transição demográfica atrasado é relevante o investimento em políticas educacionais que absorvam a população que ainda ingressará nas redes de ensino. Dado ainda que o crescimento populacional ocorra, principalmente, na parcela com menor nível de renda, políticas de controle de natalidade e de saúde são relevantes para amenizar os impactos negativos sobre sociedade.

\section{REFERÊNCIAS}

BANDEIRA, Mario Leston. Teorias da População e modernidade: o caso português. Análise Social, v. 31, p. 7-43, 1996. Disponível em: http://analisesocial.ics.ul.pt/docu mentos/1223389966U6olN8wu5Rt70DI3.pd f. Acesso em: 10 nov. 2016.

BECKER, Fernanda. Demografia e educação no Brasil: as desigualdades regionais. In: XV Encontro Nacional de Estudos populacionais, 2006. Anais ... Caxambu, MG: ABEP, 2006.

BECKER, Gary Stanley. A Treatise on the Family. Harvard University Press, 1993. Disponível

em:

https://brunofvieira.files.word

press.com/2012/12/gary-becker-a-treatiseon-the-family.pdf. Acesso em: ago. 2016.

BLOOM, David E.; CANNING, David; SEVILLA, Jaypee. Economic Growth and the demographic transition. Cambridge, NBER Working Paper Series, Working Paper 8685, 2001. Disponível em: https://pdfs.semantics cholar.org/b505/df708ce5eb076a51df7e9e 3aed9f33d8e03c.pdf. Acesso em: abr. 2017. BRITO, Fausto. A transição demográfica no Brasil: as possibilidades e os desafios para a economia e a sociedade. Texto para Discussão n. 318. UFMG/Cedeplar, 2007.

- Transição demográfica e desigualdades sociais no Brasil. Revista brasileira de Estudos da População, v. 25, n. 1, São Paulo, p. 5-26, 2008.

CALDWELL, John Curtis. Demographic Theory: A Long View. Population and Development Review, v. 30, n. 2, p. 297-316, 2004. DATASUS. Departamento de Informática do SUS. Estatísticas Vitais. Disponível em: <http://datasus.saude.gov.br/informacoesde-saude.> Acesso em: 15 nov. 2016.

FÁVERO, Luis Paulo Lopes. Análise de dados: modelagem multivariada para tomada de decisões. Rio de Janeiro: Elsevier, 2009. GALOR, Oded; WEIL, David N. Population, Technology, and Growth: From Malthusian Stagnation to the Demographic Transition and Beyond. The American Economic Review, v. 90, p. 806-828, 2000. Disponível em: <http://www.jstor.org/stable/117309.>.

Acesso em: 6 nov. 2016.

HAIR, Joseph; BLACK, Willian; BABIN, Barry; ANDERSON, Rolph; TATHAM, Ronald;. Análise multivariada de dados. 5. ed. Porto Alegre: Bookman, 2005.

IBGE. Censo Demográfico 2000. Ministério do Planejamento, Orçamento e Gestão. Rio de Janeiro, 2016.

. Censo Demográfico 2010. Ministério do Planejamento, Orçamento e Gestão. Rio de Janeiro, 2016.

Projeção da População por sexo e idade: 2000-2060. Revisão 2013. Ministério do Planejamento, Orçamento e Gestão. Rio de Janeiro, 2016.

KIRK, Dudley. Demographic Transition Theory. Population Studies, v. 50, n. 3, p. 361- 
387, 1996.

LIMA, João Eustáquio. Curso de Análise Estatística Multivariada. Universidade Federal de Viçosa. Minas Gerais, 2011.

MALTHUS, Thomas Robert. Princípios de economia política e considerações sobre sua aplicação prática: ensaio sobre a população. 2. ed. São Paulo: Nova Cultural LTDA, 1986.

MINGOTI, Sueli Aparecida. Análise de dados através de métodos de estatística multivariada: uma abordagem aplicada. Belo Horizonte: UMG, 2005.

NOTESTEIN, Frank Wallace. Economic problems of population change. London: Oxford University Press, 1953. Disponível em: http://prelim2009.filmbulletin.org/readings /04-Population/Notestein.pdf. Acesso em: ago. 2016.

ONU. Organização das Nações Unidas. Princípios de Empoderamento das Mulheres. Disponivel em: <http://www.onumulheres. org.br/wp-content/uploads/2016/04/carti Iha_WEPs_2016.pdf>. Acesso em: 20 nov. 2016.

PAIVA, Paulo de Tarso Almeida; WAJNMAN, Simone. Das causas às consequências econômicas da transição Demográfica no Brasil. Revista Brasileira de Estudos de População, v.22, 2005.

PNUD - PROGRAMA DAS NAÇÕES UNIDAS PARA O DESENVOLVIMENTO. Atlas do Desenvolvimento Humano no Brasil. 2013. Disponível em: http://www.atlasbrasil. org.br/2013/pt/. Acesso em: ago. 2016.

SOLOW, Robert Merton. A contribution to the theory of economic growth. The Quaterly Journal of Economics, v. 70, n. 1. P. 65 - 94, 1956. Disponível em: http://pi ketty.pse.ens.fr/files/ Solow1956.pdf. Acesso em: nov. 2016.
Artigo recebido em 20 de outubro de 2017. Aprovado em 29 de novembro de 2017. 